\title{
The Role of Cognitive-Behavioural Therapy for Patients with Depression in Parkinson's Disease
}

\author{
Andreas Charidimou, ${ }^{1}$ John Seamons, ${ }^{1}$ Caroline Selai, ${ }^{1}$ and Anette Schrag ${ }^{2}$ \\ ${ }^{1}$ Institute of Neurology, The National Hospital for Neurology and Neurosurgery, University College London (UCL), \\ Queen Square, London WC1N 3BG, UK \\ ${ }^{2}$ Department of Clinical Neurosciences, Institute of Neurology, University College London, Royal Free Campus, \\ London NW3 2PF, UK \\ Correspondence should be addressed to Anette Schrag, a.schrag@medsch.ucl.ac.uk
}

Received 7 December 2010; Accepted 25 February 2011

Academic Editor: Dag Aarsland

Copyright () 2011 Andreas Charidimou et al. This is an open access article distributed under the Creative Commons Attribution License, which permits unrestricted use, distribution, and reproduction in any medium, provided the original work is properly cited.

\begin{abstract}
Depression is a common complication of Parkinson's disease (PD) with considerable impact on patients' quality of life. However, at present the most appropriate treatment approach is unclear. There are limited data on antidepressant medications in PD-associated depression ( $\mathrm{CPD}$ ) and those available suggest limited efficacy and tolerability of these drugs. Cognitive behavioural therapy (CBT) has been shown to be an effective treatment of depressive disorders. Treatment of dPD with CBT may pose particular challenges, including possible different pathophysiology, physical and mental comorbidities, and barriers to treatment through disability, which do not allow simple transfer of these results to patients with dPD. However, a number of case reports, case series, and small pilot studies suggest that this is a promising treatment for patients with PD. We here summarise the published evidence on this treatment in dPD.
\end{abstract}

\section{Introduction}

Although Parkinson's disease (PD) is diagnosed on the basis of the classic features of motor disturbance $[1,2]$, it is now widely recognised that nonmotor symptoms are common, and occur across all stages of the disease [3]. Depressive disorders affect approximately $40 \%$ of patients with $\mathrm{PD}$ [4-7]. They are linked to functional impairment, cognitive decline, and faster disease progression and are the main determinant for poor quality of life in PD [8-12]. They are also associated with increased health care costs in the population, raising both direct and indirect costs $[13,14]$.

Despite this established negative impact, depressive symptoms in PD are underrecognised and undertreated in clinical practice [15]. Additionally, there is a lack of welldesigned studies that can guide clinical management of these patients. So far, only a few double-blind, placebo-controlled trials have specifically assessed antidepressant use for PD patients $[16,17]$, and even fewer research data exist on nonpharmacological approaches for PD-associated depression $[18,19]$. As a result, evidence-based recommendations and consensus on the best treatment choice for this patient population are scarce [20].

The previous pharmacological studies have shown that medication traditionally used for depression in older people (e.g., SSRIs) may not be more effective than placebo in PD, or may be difficult to utilise in this age group due to the aggravation of orthostatic hypotension, constipation, and cognitive impairment (e.g., tricyclic antidepressants) [2123]. In addition, depressive symptoms in PD often do not fulfil the criteria for major depression, and it may therefore not be appropriate to treat such cases with medication.

Cognitive behaviour therapy (CBT) is well established for the treatment of depression, and there is considerable evidence from depression without PD that CBT in this patient group is effective. This has recently been shown to include older patients [24]. However, it is recognised that $\mathrm{dPD}$ may differ from depression without PD, both 
in terms of aetiology and patient characteristics. Patients with PD are typically older, have physical impairment and subtle cognitive deficits, and therefore delivery, feasibility, and outcome of CBT may be different in patients with PD.

In this paper we consider the role of cognitive-behavioural therapy (CBT) as a potential approach for patients with $\mathrm{PD}$ and associated depression, based on a systematic review of published studies in the area.

\section{Methods: Scope of This Review, Search Strategy, and Selection Criteria}

We included all types of studies of CBT for depressive symptoms in PD patients (including single case studies, case series, and pilot studies of any duration), and systematically reviewed the evidence for effectiveness of CBT in $\mathrm{dPD}$.

References for this review were identified by searching PubMed for the last 40 years using the search terms "Parkinson's disease" or "Parkinsonism" or "Parkinsonian" and "Depression" or "affective disorders" or "mood disorders"; these results were cross-referenced with the search terms "CBT" or "cognitive therapy" or "psychotherapy". In addition, the following databases were searched using the same strategy: MEDLINE (1950 to November 2010), EMBASE (1980 to November 2010), EMBASE Classic (1947 to 1979), PsychINFO (1806 to November 2010), PsycEXTRA (1908 to November 2010), PsycBOOKS (1806 to November 2010), Web of Science (January 1981 to November 2010), and AMED (January 1985 to November 2010). All abstracts were read, and the articles found were further screened and selected as follows: only articles in the English language were selected, and only those articles reporting data on adults using cognitive or cognitive behavioural therapy for $\mathrm{dPD}$. We also searched the Cochrane Library for systematic reviews. The reference lists of the selected articles with the above strategy were checked for additional materials when appropriate. Hand searching of neurologic, psychiatric, and related literature was also performed. Moreover, online resources were searched systematically, including Medscape, NICE clinical guidelines, Department of Health publications, and registers of upcoming trials.

All the selected studies were assessed for their methodological quality. Key areas of evaluation included the accurateness/robustness of the diagnoses of depressive symptoms and idiopathic PD, the outcome measures used and their validity in $\mathrm{PD}$ and $\mathrm{PD}$ depression (with a special attention on the rating scales for evaluating changes in depression severity), the accurate description of the intervention used, and quantification of cognitive impairment and other comorbidities.

\section{Results}

We screened 1579 article abstracts in total; the search yielded a number of review articles and 15 studies of CBT for depression in PD patients. Of these, 2 were single case studies, and the rest were cases series and pilot studies, which are depicted in Table 1 and presented and discussed in the rest of this review.
3.1. Pilot Studies and Case Series. Dreisig et al. [25] reported a pilot study to explore the effects of CBT in 9 patients with PD compared to 70 matched control subjects who received treatment as usual. The 3-month CBT program included self-help and individual sessions. After this period, based on the Psychological Profile Questionnaire (including depression scores), the group receiving CBT showed significantly more improvement $(P<.01)$ compared to the control group. However, this study was not aimed specifically at depression in PD. Overall, the percentage of moderate or major depression amongst PD patients was low $(\sim 10 \%)$, and no PD patients in the CBT group had major depression. Methodological problems also include lack of randomisation, small sample size, and choice of instrument (not a validated depression scale). The study was also limited to young PD patients, which were self-referred, which could have led to selection bias. It is also important to note that the content of CBT was not described in detail making evaluation difficult. Nevertheless, the study by Dreisig et al. provided data in a small series of patients in a naturalistic setting with some control data and suggests that CBT might be effective for patients with PD.

Dobkin et al. explored the feasibility of using CBT to treat depression in an open study in 15 PD patients [26]. All patients had major depression based on DSM-IV and no evidence of dementia, psychosis, or significant motor fluctuations. The content and delivery of treatment was developed via a previous case series [35]. Patients received 10-14 sessions of personalized CBT incorporating relaxation techniques and sleep hygiene education. Treatment delivery was modified to aid memory retention, and caregivers attended separate educational sessions. The Hamilton Rating Scale for Depression (HAM-D) and the Beck Depression Inventory (BDI) were used as the primary outcome measures, while negative thoughts (Inference Questionnaire; IQ), anxiety (State Trait Anxiety Inventory; STAI), and perception of social support (Adaptive Inferential Feedback Questionnaire, AIFQ) were evaluated as secondary outcome measures. Of the 13 patients who completed the study, $80 \%$ experienced significant reductions in depressive symptoms (HAM-D; BDI, $P<.0001$ ). A significant decrease was also noted in negative inferences (IQ, $P<.001$ ), while the perception of social support was increased significantly (AIFQ, $P<.001$ ). Despite the methodological limitations of this study (lack of control group, small sample size, and no formal assessment of the contribution of caregivers), the detailed study design, inclusion of qualitative methods, involvement of caregivers, exclusion of patients with motor fluctuations and the delivery of treatment by a trained CBT therapist, provide good preliminary evidence as to the feasibility and potential effectiveness of this treatment modality for $\mathrm{dPD}$.

Another uncontrolled study of CBT in targeting depression within the context of PD was recently reported recently by Farabaugh et al. [27]. Eight depressed patients were enrolled into an open study of 12-week individually tailored CBT treatment. The authors reported a significant linear decrease in mean Hamilton Rating Scale for Depression (HAM-D) scores over the treatment period. At the study 
TABLE 1: Summary of studies reviewed focusing on CBT for patients with PD and depression.

\begin{tabular}{|c|c|c|c|c|}
\hline Study & Sample size & Intervention & Delivery & Outcome measures \\
\hline Dreisig et al. [25]* & 79 PD: 9 CBT; 70 control & Self-help \& tailored CBT & Author (trained) & $\begin{array}{l}\text { Psychological Profile } \\
\text { Questionnaire, SDS }\end{array}$ \\
\hline Dobkin et al. [26] & $15 \mathrm{PD} ; 15$ caregivers & $\begin{array}{l}\text { Tailored CBT \& } \\
\text { caregiver program }\end{array}$ & Clinical psychologist & $\begin{array}{l}\text { HAM-D, BDI, IQ, STAI, } \\
\text { AIFQ }\end{array}$ \\
\hline Farabaugh et al. [27] & $8 \mathrm{PD}$ & $\begin{array}{l}\text { Individual CBT } \\
\text { treatment }\end{array}$ & Author (trained) & HAM-D \\
\hline Veazey et al. [28]* & $\begin{array}{l}10 \mathrm{PD} \text {; randomized into } \\
\text { CBT group or support } \\
\text { group (control) }\end{array}$ & $\begin{array}{l}\text { Telephone-administered } \\
\text { CBT }\end{array}$ & Author (trained) & $\begin{array}{l}\text { MMSE, PHQ-9, BAI, } \\
\text { PDQ-39, SCID }\end{array}$ \\
\hline Leroi and King [29] & $8 \mathrm{PD}$ & $\begin{array}{l}\text { Individual CBT } \\
\text { treatment }\end{array}$ & $\begin{array}{l}\text { Consultant clinical } \\
\text { psychologist }\end{array}$ & GAS, unreported scales \\
\hline Simons et al. [30] & 22 PD; 14 Caregiver & $\begin{array}{l}\text { Educational program } \\
\text { with CBT elements }\end{array}$ & Not reported & $\begin{array}{l}\text { SDS, PDQ39, participant } \\
\text { evaluation }\end{array}$ \\
\hline A'Campo et al. [31] & 64 PD; 46 Caregiver & $\begin{array}{l}\text { Educational program } \\
\text { with CBT elements }\end{array}$ & Not reported & $\begin{array}{l}\text { Dutch version of PDQ-39 } \\
\text { and EQ-5D, SDS, VAS }\end{array}$ \\
\hline Macht el al. [32] & $151 \mathrm{PD}$ & $\begin{array}{l}\text { Educational program } \\
\text { with CBT elements }\end{array}$ & Author (trained) & $\begin{array}{l}\text { Patients' ratings of the } \\
\text { comprehensibility and } \\
\text { feasibility of the } \\
\text { programme as well as } \\
\text { mood ratings before and } \\
\text { after each session }\end{array}$ \\
\hline Cole and Vaughan [33] & $5 \mathrm{PD}$ & CBT & Author (trained) & BDI-II, GDS15, PDQL \\
\hline Feeney et al. [34] & $4 \mathrm{PD}$ & CBT & Not reported & BDI-II \\
\hline Dobkin et al. [35] & $3 \mathrm{PD} ; 3$ caregivers & $\begin{array}{l}\text { Tailored CBT \& } \\
\text { caregiver program }\end{array}$ & Not reported & $\begin{array}{l}\text { HAM-D, BDI, IQ, STAI, } \\
\text { AIFQ }\end{array}$ \\
\hline Macht et al. [36] & $3 \mathrm{PD}$ & Tailored CBT & Author (trained) & Clinician’s Assessment \\
\hline Fitzpatrick et al. [37] & $12 \mathrm{PD}$ & MBCT & Author & $\begin{array}{l}\text { Not formal outcome } \\
\text { measures; Interpretative } \\
\text { phenomenological analysis }\end{array}$ \\
\hline Gupta and Bhatia [38] & $1 \mathrm{PD}$ & CBT & Author (trained) & Clinician's Assessment \\
\hline Laidlaw et al. [39] & $1 \mathrm{PD}$ & CBT & Author (trained) & Clinician's Assessment \\
\hline
\end{tabular}

${ }^{*}$ Studies with a control group.

AIFQ: Adaptive Inferential Feedback Questionnaire; BDI: Beck Depression Inventory; CBT: Cognitive Behavioural Therapy; CES-D: Centre for Epidemiologic Studies Depression Scale; GAS: Global Assessment Scale; GDS: Geriatric Depression Scale; HAM-D: Hamilton Rating Scale for Depression; HRQL: Health Related Quality of Life; IQ: Inference Questionnaire; MBCT: mindfulness-based cognitive therapy; MMSE: Mini-mental State Examination; PD: Parkinson's Disease; PDQL: Parkinson's Disease Quality of Life Questionnaire; PDQ39: Parkinson's Disease Questionnaire 39; PHQ-9: Patient Health Questionnaire9; SCID: Structured Clinical Interview for DSM-IV; SDS: Zung Self-Rating Depression Scale; STAI: Spielberger State-Trait Anxiety Inventory; VAS: Visual Analogue Scale.

endpoint more than half of the patients (57\%) met the criteria for complete remission. With the same limitations as the above studies, this pilot study also adds to the suggestion that CBT may be beneficial in this population of patients and may be an alternative or adjunct to pharmacological treatment strategies.

Veazey et al. [28] reported a controlled study of telephone-administered CBT for PD patients with depression and anxiety. At baseline, the ten patients who took part were assessed using MMSE, Patient Health Questionnaire-9 (PHQ-9), Beck Anxiety Inventory (BAI), Parkinson's Disease Questionnaire-39 (PDQ-39), and the Structured Clinical Interview for DSM-IV (SCID). Based on BAI and PHQ-9, half of the patients had anxiety and depression, 4 (40\%) had only anxiety and 1 had only depression. Study participants were randomized into either a CBT group or a support group (control). The CBT groups received 1 in-person CBT session, followed by 8 weekly telephone-delivered CBT sessions. Sessions covered depression and anxiety education, relaxation training, cognitive therapy, problem solving, activity scheduling, and sleep-management skills. The support group received 8 weekly phone calls from a therapist, during which the therapist followed a script including questions about the patient's general wellbeing; psychotherapy elements were not included. Whilst this was a pilot study on the feasibility of CBT with descriptive data, there was an improvement in depression (based on changes in PHQ-9 scores) for both groups following treatment and at 1-month followup. The CBT group was more improved, but with a small difference, compared to the support group. Similarly, anxiety 
improved for both groups, with a greater improvement in the CBT group. The study is the first to use remotedelivered CBT for PD patients and to use a control group with a nonspecific "talking therapy". Delivering CBT by telephone allows participants more flexibility in scheduling and keeping weekly appointments than in-person meetings. However, this approach also poses a number of problems; in particular, it may be more difficult to keep patients motivated and active, to ensure that patients complete homework and understand treatment sessions. Thus, it may be preferable to test the efficacy of CBT for PD-associated depression using standard therapy protocols (i.e., face-to-face) before using modified approaches with potential confounders.

In an abstract, Leroi and King [29] reported an uncontrolled pilot study to evaluate the feasibility of modified CBT for $\mathrm{PD}$ patients with depression and/or anxiety based on DSM-IV and Geriatric Depression Scale (GDS). The study included 5 patients with idiopathic PD, two of whom had major depressive disorder, one nonmajor depression, and two mixed anxiety and depression at study start. CBT (6-10 sessions) was delivered by a consultant clinical psychologist and relevant changes in depression/anxiety were measured using the Global Attainment Scale (GAS) and other unreported scales. Depression and anxiety scores improved in all participants, with $100 \%$ reaching subthreshold levels for clinical significance. The mean GAS score reflected moderate to marked improvement on pretreatment selfselected outcome goals, while the therapy was found to be well-tolerated and feasible. Conclusions from this study are difficult to draw as there were only overall 5 patients, no control group and the validity of the GAS in this patient group has not been tested. In addition, the authors reported modifications to the standard CBT protocol, but these were not detailed in the abstract.

Simons and colleagues [30] reported an eight-session group educational program, called the EduPark, that was developed to promote coping strategies and address common psychosocial problems in PD patients and caregivers. EduPark covers many of the skills included in standard CBT [36] and was evaluated in a pilot study of 36 participants. Patients completed a self-rating depression scale (SDS) before and after the program [40]. EduPark yielded shortterm improvements in visual analogue ratings of mood and was favorably evaluated by participants. However, no significant effects on quality of life or depression as assessed on the SDS were found in patients or caregivers [30]. Studies from other participating countries reported similar findings in overall 151 patients with improvement of psychosocial functioning and mood assessment on a visual analogue scale but not on the SDS [32]. The limitations of this study, in addition to the lack of control group, include uncertainty whether any of the enrolled patients had a formal diagnosis of depression at baseline. In addition, although educational programs are of great importance, by definition they are more disperse and less intensive than individually tailored CBT. Achievement of significant changes in depression may require a more intensive and/or longer period of treatment.

The two uncontrolled studies conducted during the development of EduPark [30, 32] were more tailored to evaluate its feasibility rather than its effectiveness. A'Campo et al. recently described a RCT which evaluated the effectiveness of this educational programme in PD patients and their caregivers from The Netherlands [31]. Sixty-four PD patients and 46 caregivers participated and were allocated to either the intervention group (receiving eight weekly, 90minute duration sessions) or the control group (receiving usual care). The primary outcomes measure of this trial were psychosocial burden, depression and QoL of both patients and caregivers and depression (using the SDS). Participants in both groups were also asked to rate their present mood before and after each individual session on a 100-point visual analogue scale (VAS). No significant effects on the primary outcome measures of the patients were found after the completion of the programme. A marginally significant trend of improvement was only observed in the PDQ-39, favouring the intervention group $(P=.015)$. However, based on the VAS, patients' mood was found to be significantly improved from before to after sessions $(P<$ .001). Moreover, concerning the caregivers', psychosocial problems and need for help of the intervention group were significantly improved compared to the control group. The improvements on mood as highlighted by the VAS indicate that the patients in the intervention group felt better after the session. However, this scale represents an oversimplification of mood measuring and there was no comparison to a control group. Despite the randomised controlled nature of this trial, the methodological weaknesses of this trial include the fact that the participants had no or only minimal depressive symptoms, which were assessed by a relatively insensitive instrument (SDS). Moreover, the authors do not report how the diagnosis of the depressive disorders was made in this patient population (apart from the depression rating scale score) and how PD was diagnosed.

Cole and Vaughan [33] reported a case series where brief home-based CBT was used to treat depression in the context of PD. Five patients aged 54-82 years were referred for treatment from a movement disorders clinic following a diagnosis of possible depression (based on Geriatric Depression ScaleGDS). Three out of 5 patients had Beck Depression Inventory (BDI) scores indicating major depression, and none of them had dementia. CBT was protocol driven and based on a self-help booklet. One 60-minute CBT session per week was allocated for each section of the booklet, over 7 weeks. Following treatment, there were reductions in depression scores. However, these were marginal in the patients with less severe depression and changes in depression scores were not accompanied by changes in QoL. Lack of meaningful improvement in two patients was felt to be at least partly related to intervening illness and personal factors. This study is one of the few which reported how PD was diagnosed (UK Parkinson's Disease Society Brain Bank). However, diagnosis of depressive disorder was not made based on DSM-IV criteria. It is possible that the small patient number and the short baseline durations hamper the sensitivity of detecting an effectiveness of CBT. However, the results may indicate that less severe depression may be less responsive to this intervention. 
The effects of group CBT for depression and anxiety in PD were also assessed in a small case series of 4 patients aged 56-81 years [34]. Participants were not cognitively impaired (based on Mini-mental Mental State ExaminationMMSE), three of them had major depression [41], while one had a dysthymic disorder based on the Mini-International Neuropsychiatric Interview (MINI) [42]. Minor adaptations to the delivery of CBT were made, such as a reduction on the amount of writing to account for motor difficulties. Treatment resulted in a clinically significant improvement in depression in 3 out of 4 patients according to the BDI. This case series is documenting the feasibility of CBT for patients with PD and depression with maintenance of gains at the 1-month followup. Again, the small sample size, the lack of control, and exclusion criteria make the applicability of these findings to other patients with $\mathrm{PD}$-associated depression uncertain. One other disadvantage of this particular study is that it does not report who delivered CBT, or whether a CBT protocol was used; therefore, the content of treatment is uncertain. One patient in this study had been diagnosed with PD 12 years ago, compared with 1-3 years for the rest of the participants, and this patient did not show improvement. At present it is unclear whether more advanced PD is a predictor of nonresponse to CBT.

Dobkin et al. [35] reported a case series of 3 patients with PD meeting the DSM-IV criteria for major depression (and with no evidence of dementia or psychosis). The primary outcome measure used was Hamilton Rating Scale for Depression (Ham-D). Patients received 12-14 sessions of individually tailored CBT. After identifying stressors that appeared to contribute to depressed mood, short and longterm plans were developed to minimize stress and maximize QoL by emphasing behavioural activation and problemsolving strategies around physical limitations. All patients were experiencing sleep difficulties, so sleep hygiene techniques were incorporated. In parallel, relaxation techniques, such as diaphragmatic breathing, were also incorporated to address anxiety and somatic complaints. Treatment delivery was modified when necessary to aid memory retention. Patients and caregivers evaluated positively the overall treatment program. All patients achieved $\sim 50 \%$ reduction in depression based on HAM-D and BDI, maintained at 1-month follow-up. HAM-D and BDI endpoints for two patients indicated only mild depression, and all participants demonstrated decreased negative cognitions based on IQ and BDI. The authors later reported the results of a large pilot study based on these results (see above).

Another case series of 3 self-referred PD patients treated with CBT were reported by Macht and colleagues [36]. The patients differed in age, disease duration, disease severity, and disability, whilst none showed signs of cognitive impairment. CBT was tailored to each patient via formulation, and adjunctive techniques were used, such as strategies to cope with motor fluctuations. Notably, only one patient was reported to have depressive symptoms, although their pre/posttreatment severity was not reported. The authors reported that all patients improved in their ability to cope with their disease following CBT, but this effect was not quantified. The limited information provided in this study regarding the CBT intervention, the patient group and aim of the study (improving coping with PD), combined with the small sample size make it difficult draw conclusions regarding the effect of CBT of $\mathrm{dPD}$ in this population.

An exploratory study was recently reported by Fitzpatrick and colleagues which analysed the experiences of 12 PD patients after an 8-week course of mindfulness-based cognitive therapy (MBCT) [37]. This type of psychological therapy combines elements of both cognitive therapy and mindfulness technique originating from Eastern spiritual and philosophical tradition. Mindfulness prioritizes learning how to pay attention on purpose, in the present moment and without judgement. In this framework MBCT is built around accepting thoughts and feelings in a nonjudgemental way with the aim of correcting cognitive distortions (rather than trying to suppress and avoid them) [43]. The authors of this study made it clear that they did not intend to assess the effectiveness of MBCT using formal outcome measures, but to gather preliminary evidence of its acceptance and perceived helpfulness from patients with PD [37]. Interpretative phenomenological analysis was the qualitative approach chosen for the analysis of emerging themes in this study (summarised from author's transcripts, compared against all cases, and also analysed by a second author). The analysis of both pre- and postcourse interviews, yielded 4 major themes including changing patterns of coping, the role of this intervention in consolidating already existing coping skills in the context of loss, the importance of group support effects and the dualism of experience between PD and mindful meditation. The results of this study should be evaluated under the framework of its original purposes, design, and methodology. Overall, it demonstrated the acceptability of MBCT in a group of patients with PD (based on the positive/supportive informal feedback from the participants and the well attendance of subsequent "catchup" groups). Moreover, this study analysed in depth what were the key factors making this course successful. Unfortunately, there is little information of the depressive symptomatology of the participants. Nine patients were found to have clinical levels of distress in one or more of the subscales of depression, anxiety, and stress evaluated by the Depression Anxiety and Stress Scales (DASS). This is the first study of MBCT in the context of PD suggesting this intervention may be useful in future intervention studies, and the emerging themes of this intervention can also help understand and enhance patterns of coping with PD.

3.2. Case Studies. Gupta and Bhatia [38] treated a 90-yearold PD patient with depression and suicidal ideation using home-based CBT. The focus of CBT was in reinforcing the patient's positive interactions with his daughter (the caregiver) and increasing his participation in enjoyable activities. CBT indeed decreased the patient's depressive symptoms, improved interactions with his caregiver, and increased activity levels. Laidlaw et al. [39] reported the effect of CBT in a patient with anxiety, depression, and insomnia following his diagnosis with PD. The patient had marked resting tremor in his right hand, reduced pleasurable activities and he had stopped going out unaccompanied. 
Antidepressant medication had little effect; in contrast CBT reduced depression, anxiety, and insomnia, but the largest gains were in activity levels. The authors emphasise the importance of making interventions addressing the specific difficulties of the patients. For example, the patient in this paper became embarrassed and his mood dropped when his tremor prevented him from performing motor tasks. By the end of treatment, the patient was more willing to confront embarrassing situations and use CBT techniques to challenge negative thought patterns [39].

\section{Discussion}

We identified and described 15 studies on the application of CBT in depressed PD patients. The majority of the studies reported functionally important improvements using this treatment strategy to target depressive symptoms in patients with PD, some with large effect sizes. Whilst these data are encouraging, at present there are no large randomised control trials (RCTs) in this area and the largest study included overall 15 patients (excluding the studies using education programmes rather than CBT ipse) [26]. Due to the small patient sample sizes, the results from these pilot studies and case series may overstate the effect of this intervention. Conclusions from these trials therefore have to be cautious, particularly given the large placebo effects known to exist in both depression and PD. Interpretation of the above studies should also take into account the following considerations.

The evaluation of CBT studies poses particular challenges. CBT is not a single, standard form of therapy, but, whilst including a core CBT strategy, incorporates a collection of different psychotherapeutic approaches and components. This makes it difficult to compare different studies, as they may have used different CBT approaches. In addition, many published studies do not provide adequate details of the CBT intervention complicating comparison further. For example, the intervention may not have contained the same noncore aspects of CBT; and treatment outcomes may be attributable to adjunctive techniques such as exercise or relaxation. However, despite this variation, all studies focusing on the application of CBT incorporate the same core elements. CBT is defined as a form of therapy that focuses on the relationship between thoughts, feelings, and behaviours. As such, even with many variations in its application from person to person, CBT is distinct and very distinguishable from other psychotherapeutic approaches (e.g., interpersonal, psychodynamic, etc.). In this context, the aspects and elements of CBT that are most beneficial are likely to vary for each individual patient.

Thus, treatment is typically tailored to patients. In clinical practice, CBT often uses protocols which have been developed for specific disorders (e.g., depression, anxiety, etc.). However, the first stage of CBT is patient assessment and the development of a formulation-a working model of the patient's problems. How the patient is treated, and the selection of therapeutic interventions, depends upon this formulation. Formulation allows CBT theory to be applied to unique individuals with distinct patterns of thoughts and emotions. In this manner, CBT may be based on a protocol, but it is tailored to each patient. As new information emerges during treatment the formulation continues to evolve. The formulation informs the selection of specific cognitive, behavioural, and physical interventions. Whilst this introduces a certain variability, this is also one of the advantages of CBT. Other advantages of CBT include its structured, time-limited, and goal-oriented approach, the lack of medication side effects and long-term effects even after the treatment has been completed $[44,45]$.

Further methodological considerations in evaluation of the available data include the variation in reporting PD diagnosis and few studies used the UK Parkinson's Disease Society Brain Bank or other standardized criteria. In addition, current studies are likely to have been included in highly motivated, cognitively relatively intact and less disabled patients. However, it is currently unclear whether and to what extent severity of PD or other disease variables such as mild cognitive impairment influence the feasibility or effectiveness of this intervention, and whether this treatment approach is suitable across disease stages. Several studies report excluding patients with diagnoses of dementia, but do not report levels of cognitive impairment [26, 33, 35]. It is possible that subtle cognitive impairments could affect treatment outcomes. However, the exclusion of cognitively impaired patients also limits the applicability of studies to PD populations, where rates of cognitive impairment are high. A further important consideration is the inclusion of motor fluctuations. Only one study excluded patients with motor fluctuations [26]. "Off" periods are associated with acute episodes of depression and anxiety and could therefore confound treatment outcomes [46]. If not excluded, patients with motor fluctuations should be assessed during "on" periods [47].

Whilst diagnosis of depression in PD patients was generally made based on standard criteria (with their limitations for use in PD) rather than rating scales, severity varied and a wide range of depression rating scales were used as outcome measures in the different studies. Given the significant symptom overlap between depression, parkinsonism, and apathy in these patients, it is currently unclear which scale is the best choice in a trial of CBT in APD [47]. However, given that CBT is unlikely to influence underlying parkinsonism, this treatment is less likely than some medications to improve depression rating scale scores through improvement of nondepression features of PD. At present it is also unclear whether CBT may be useful for major depression or depressive disorders not fulfilling these criteria.

\section{Conclusions}

In summary, whilst additional data from large RCTs are needed to establish the efficacy of CBT in the management of depression in patients with $\mathrm{PD}$, the available evidence, even with the above limitations, is encouraging for the effectiveness of CBT as an option in this patient population. In addition to establishing feasibility and efficacy of CBT in $\mathrm{dPD}$ in large RCTs, there are a number of questions remaining to be unanswered in future studies, including to 
what extent the results of these studies can be translated and applied to the PD patients with cognitive impairment, whether this type of treatment is feasible in patients with motor and nonmotor complications, how to best assess outcome, patients of what severity of depression should be included and whether CBT needs to be modified for patients with PD. If shown to be effective in large RCTs, CBT could potentially provide a better possible nonpharmacologic treating option for clinicians for the treatment of depression in $\mathrm{PD}$, either when pharmacotherapy fails, in combination or as an alternative to pharmacological treatment of $\mathrm{dPD}$.

\section{Conflict of Interests}

The authors declare that there is no conflict of interests.

\section{Acknowledgment}

A. Charidimou and J. Seamons are joint first authors, and they have contributed equally to this paper.

\section{References}

[1] I. Litvan, K. P. Bhatia, D. J. Burn et al., "Movement Disorders Society Scientific Issues Committee report: SIC Task Force appraisal of clinical diagnostic criteria for Parkinsonian disorders," Movement Disorders, vol. 18, no. 5, pp. 467-486, 2003.

[2] D. M. Huse, K. Schulman, L. Orsini, J. Castelli-Haley, S. Kennedy, and G. Lenhart, "Burden of illness in Parkinson's disease," Movement Disorders, vol. 20, no. 11, pp. 1449-1454, 2005.

[3] K. R. Chaudhuri and A. H. Schapira, "Non-motor symptoms of Parkinson's disease: dopaminergic pathophysiology and treatment," The Lancet Neurology, vol. 8, no. 5, pp. 464-474, 2009.

[4] J. R. Slaughter, K. A. Slaughter, D. Nichols, S. E. Holmes, and M. P. Martens, "Prevalence, clinical manifestations, etiology, and treatment of depression in Parkinson's disease," Journal of Neuropsychiatry and Clinical Neurosciences, vol. 13, no. 2, pp. 187-196, 2001.

[5] W. M. McDonald, I. H. Richard, and M. R. DeLong, "Prevalence, etiology, and treatment of depression in Parkinson's disease," Biological Psychiatry, vol. 54, no. 3, pp. 363-375, 2003.

[6] A. F. G. Leentjens, "Depression in Parkinson's disease: conceptual issues and clinical challenges," Journal of Geriatric Psychiatry and Neurology, vol. 17, no. 3, pp. 120-126, 2004.

[7] J. S. Reijnders, U. Ehrt, W. E. Weber, D. Aarsland, and A. F. G. Leentjens, "A systematic review of prevalence studies of depression in Parkinson's disease," Movement Disorders, vol. 23, no. 2, pp. 183-189, 2008.

[8] A. Schrag, M. Jahanshahi, and N. Quinn, "What contributes to quality of life in patients with Parkinson's disease?" Journal of Neurology, Neurosurgery and Psychiatry, vol. 69, no. 3, pp. 308-312, 2000.

[9] S. E. Soh, M. E. Morris, and J. L. McGinley, "Determinants of health-related quality of life in Parkinson's disease: a systematic review," Parkinsonism \& Related Disorders, vol. 17, no. 1, pp. 1-9, 2011.
[10] A. Schrag, "Quality of life and depression in Parkinson's disease," Journal of the Neurological Sciences, vol. 248, no. 1-2, pp. 151-157, 2006.

[11] N. Pankratz, K. S. Marder, C. A. Halter et al., "Clinical correlates of depressive symptoms in familial Parkinson's disease," Movement Disorders, vol. 23, no. 15, pp. 2216-2223, 2008.

[12] J. L. Cummings, "Depression and Parkinson's disease: a review," American Journal of Psychiatry, vol. 149, no. 4, pp. 443-454, 1992.

[13] P. Berto, D. D’llario, P. Ruffo, R. Di Virgilio, and F. Rizzo, "Depression: cost-of-illness studies in the international literature, a review," The Journal of Mental Health Policy and Economics, vol. 3, no. 1, pp. 3-10, 2000.

[14] P. Sobocki, I. Lekander, F. Borgström, O. Ström, and B. Runeson, "The economic burden of depression in Sweden from 1997 to 2005," European Psychiatry, vol. 22, no. 3, pp. 146-152, 2007.

[15] A. Althaus, O. A. Becker, A. Spottke et al., "Frequency and treatment of depressive symptoms in a Parkinson's disease registry," Parkinsonism and Related Disorders, vol. 14, no. 8, pp. 626-632, 2008.

[16] M. S. Okun and H. H. Fernandez, "Will tricyclic antidepressants make a comeback for depressed Parkinson disease patients?" Neurology, vol. 72, no. 10, pp. 868-869, 2009.

[17] H. H. Fernandez and M. Merello, "Pramipexole for depression and motor symptoms in Parkinson's disease: can we kill two birds with one stone?" The Lancet Neurology, vol. 9, no. 6, pp. 556-557, 2010.

[18] R. D. Dobkin, M. Menza, and K. L. Bienfait, "CBT for the treatment of depression in Parkinson's disease: a promising nonpharmacological approach," Expert Review of Neurotherapeutics, vol. 8, no. 1, pp. 27-35, 2008.

[19] E. Sproesser, M. A. Viana, E. M. A. B. Quagliato, and E. A. P. de Souza, "The effect of psychotherapy in patients with PD: a controlled study," Parkinsonism and Related Disorders, vol. 16, no. 4, pp. 298-300, 2010.

[20] S. Ghazi-Noori, T. H. Chung, K. Deane, H. E. Rickards, and C. E. Clarke, "Therapies for depression in Parkinson's disease," Cochrane Database of Systematic Reviews, vol. 3, Article ID CD003465, 2003.

[21] D. Weintraub, K. H. Morales, P. J. Moberg et al., "Antidepressant studies in Parkinson's disease: a review and metaanalysis," Movement Disorders, vol. 20, no. 9, pp. 1161-1169, 2005.

[22] J. M. Miyasaki, "New practice parameters in Parkinson's disease," Nature Clinical Practice Neurology, vol. 2, no. 12, pp. 638-639, 2006.

[23] M. Menza, R. D. Dobkin, H. Marin et al., "A controlled trial of antidepressants in patients with Parkinson disease and depression," Neurology, vol. 72, no. 10, pp. 886-892, 2009.

[24] M. A. Serfaty, D. Haworth, M. Blanchard, M. Buszewicz, S. Murad, and M. King, "Clinical effectiveness of individual cognitive behavioral therapy for depressed older people in primary care: a randomized controlled trial," Archives of General Psychiatry, vol. 66, no. 12, pp. 1332-1340, 2009.

[25] H. Dreisig, L. Wermuth, S. Skovlund, and P. Bech, "Psychologic effects of structured cognitive psychotherapy in young patients with Parkinson disease: a pilot study," Nordic Journal of Psychiatry, vol. 53, no. 3, pp. 217-221, 1999.

[26] R. D. Dobkin, L. A. Allen, and M. Menza, "Cognitivebehavioral therapy for depression in Parkinson's disease: a pilot study," Movement Disorders, vol. 22, no. 7, pp. 946-952, 2007. 
[27] A. Farabaugh, J. J. Locascio, L. Yap et al., "Cognitivebehavioral therapy for patients with Parkinson's disease and comorbid major depressive disorder," Psychosomatics, vol. 51, no. 2, pp. 124-129, 2010.

[28] C. Veazey, K. F. Cook, M. Stanley, E. C. Lai, and M. E. Kunik, "Telephone-administered cognitive behavioral therapy: a case study of anxiety and depression in Parkinson's disease," Journal of Clinical Psychology in Medical Settings, vol. 16, no. 3, pp. 243-253, 2009.

[29] I. Leroi and P. King, "Cognitive behaviour therapy for anxiety and depression in Parkinson's disease: a pilot study," The Movement Disorder Society, vol. 23, supplement 1, p. 276, 2008.

[30] G. Simons, S. B. Thompson, and M. C. Smith Pasqualini, "An innovative education programme for people with Parkinson's disease and their carers," Parkinsonism and Related Disorders, vol. 12, no. 8, pp. 478-485, 2006.

[31] L. E. A'Campo, E. M. Wekking, N. G. Spliethoff-Kamminga, S. Le Cessie, and R. A. Roos, "The benefits of a standardized patient education program for patients with Parkinson's disease and their caregivers," Parkinsonism Relat Disord, vol. 16, no. 2, pp. 89-95, 2010.

[32] M. Macht, C. Gerlich, H. Ellgring et al., "Patient education in Parkinson's disease: formative evaluation of a standardized programme in seven European countries," Patient Education and Counseling, vol. 65, no. 2, pp. 245-252, 2007.

[33] K. Cole and F. L. Vaughan, "Brief cognitive behavioural therapy for depression associated with Parkinson's disease: a single case series," Behavioural and Cognitive Psychotherapy, vol. 33, no. 1, pp. 89-102, 2005.

[34] F. Feeney, S. Egan, and N. Gasson, "Treatment of depression and anxiety in Parkinson's disease: a pilot study using group cognitive behavioural therapy," Clinical Psychologist, vol. 9, no. 1, pp. 31-38, 2005.

[35] R. D. Dobkin, L. A. Allen, and M. Menza, "A cognitivebehavioral treatment package for depression in Parkinson's disease," Psychosomatics, vol. 47, no. 3, pp. 259-263, 2006.

[36] M. Macht, M. S. Pasqualini, and P. Taba, "Cognitivebehavioral strategies for Parkinson's disease: a report of three cases," Journal of Clinical Psychology in Medical Settings, vol. 14, no. 2, pp. 165-176, 2007.

[37] L. Fitzpatrick, J. Simpson, and A. Smith, "A qualitative analysis of mindfulness-based cognitive therapy (MBCT) in Parkinson's disease," Psychology and Psychotherapy, vol. 83, part 2, pp. 179-192, 2010.

[38] A. Gupta and S. Bhatia, "Psychological functioning in patients with Parkinson's disease," Parkinsonism and Related Disorders, vol. 6, no. 3, pp. 185-190, 2000.

[39] K. Laidlaw, L. W. Thompson, L. Dick-Siskin, and D. GallagherThompson, Cognitive Behaviour Therapy with Older People, John Wiley \& Sons, Chichester, UK, 2003.

[40] W. W. Zung, "A self-rating depression scale," Archives of General Psychiatry, vol. 12, pp. 63-70, 1965.

[41] H. Christensen, K. M. Griffiths, A. E. Korten, K. Brittliffe, and C. Groves, "A comparison of changes in anxiety and depression symptoms of spontaneous users and trial participants of a cognitive behavior therapy website," Journal of Medical Internet Research, vol. 6, no. 4, p. e46, 2004.

[42] D. V. Sheehan, Y. Lecrubier, K. H. Sheehan et al., "The Mini-International Neuropsychiatric Interview (M.I.N.I.): the development and validation of a structured diagnostic psychiatric interview for DSM-IV and ICD-10," Journal of Clinical Psychiatry, vol. 59, supplement 20, pp. 22-33, 1998.
[43] D. J. Stein, V. Ives-Deliperi, and K. G. F. Thomas, "Psychobiology of mindfulness," CNS Spectrums, vol. 13, no. 9, pp. 752756, 2008.

[44] S. D. Hollon, R. C. Shelton, and D. D. Davis, "Cognitive therapy for depression: conceptual issues and clinical efficacy," Journal of Consulting and Clinical Psychology, vol. 61, no. 2, pp. 270-275, 1993.

[45] J. Scott, "Cognitive therapy of affective disorders: a review," Journal of Affective Disorders, vol. 37, no. 1, pp. 1-11, 1996.

[46] H. Nissenbaum, N. P. Quinn, R. G. Brown, B. Toone, A. M. Gotham, and C. D. Marsden, "Mood swings associated with the 'on-off' phenomenon in Parkinson's disease," Psychological Medicine, vol. 17, no. 4, pp. 899-904, 1987.

[47] A. Schrag, P. Barone, R. G. Brown et al., "Depression rating scales in Parkinson's disease: critique and recommendations," Movement Disorders, vol. 22, no. 8, pp. 1077-1092, 2007. 


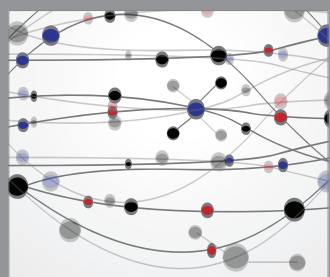

The Scientific World Journal
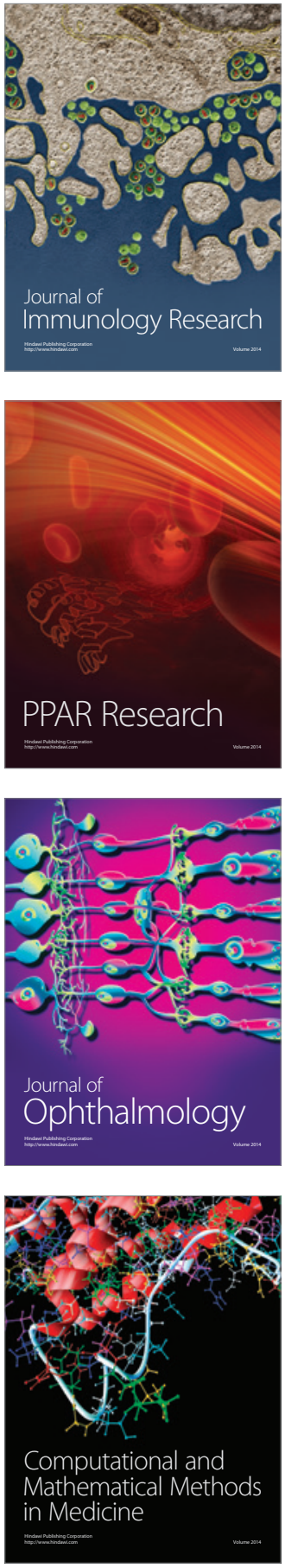

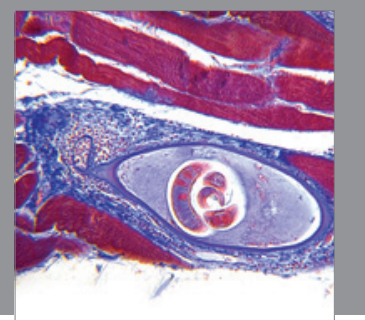

Gastroenterology

Research and Practice
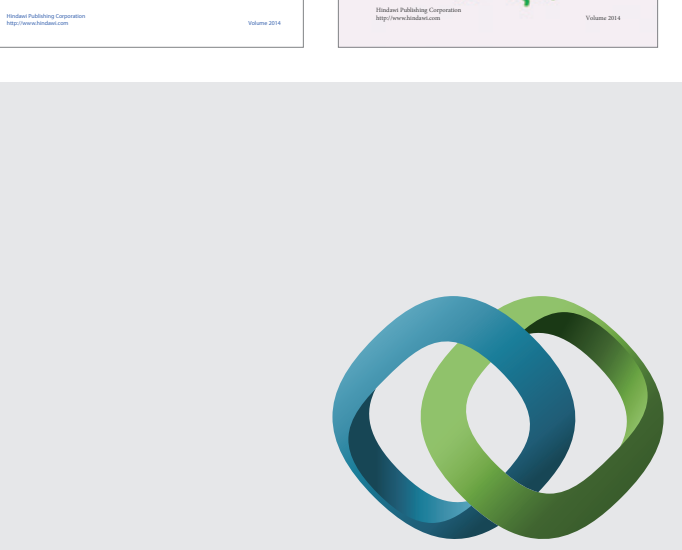

\section{Hindawi}

Submit your manuscripts at

http://www.hindawi.com
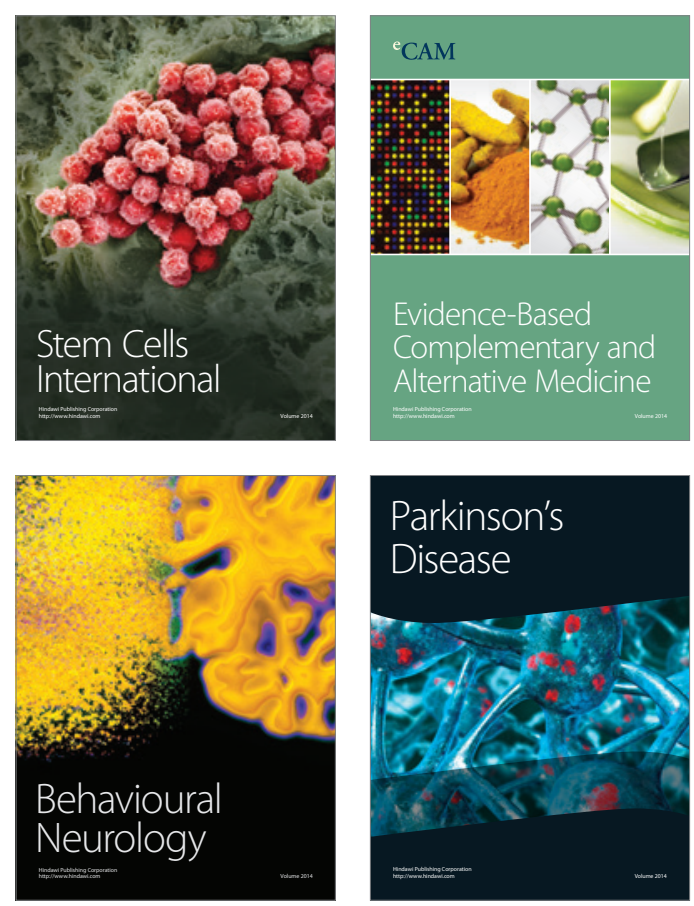

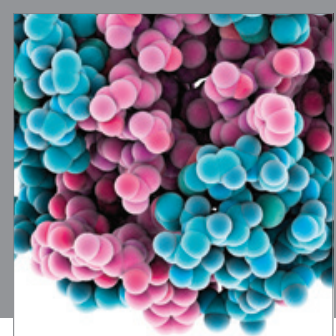

Journal of
Diabetes Research

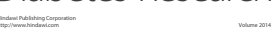

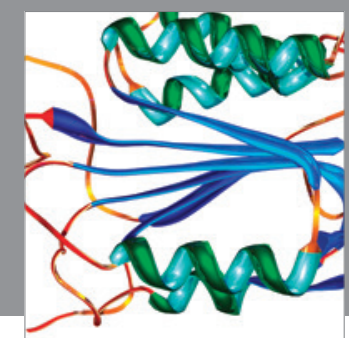

Disease Markers
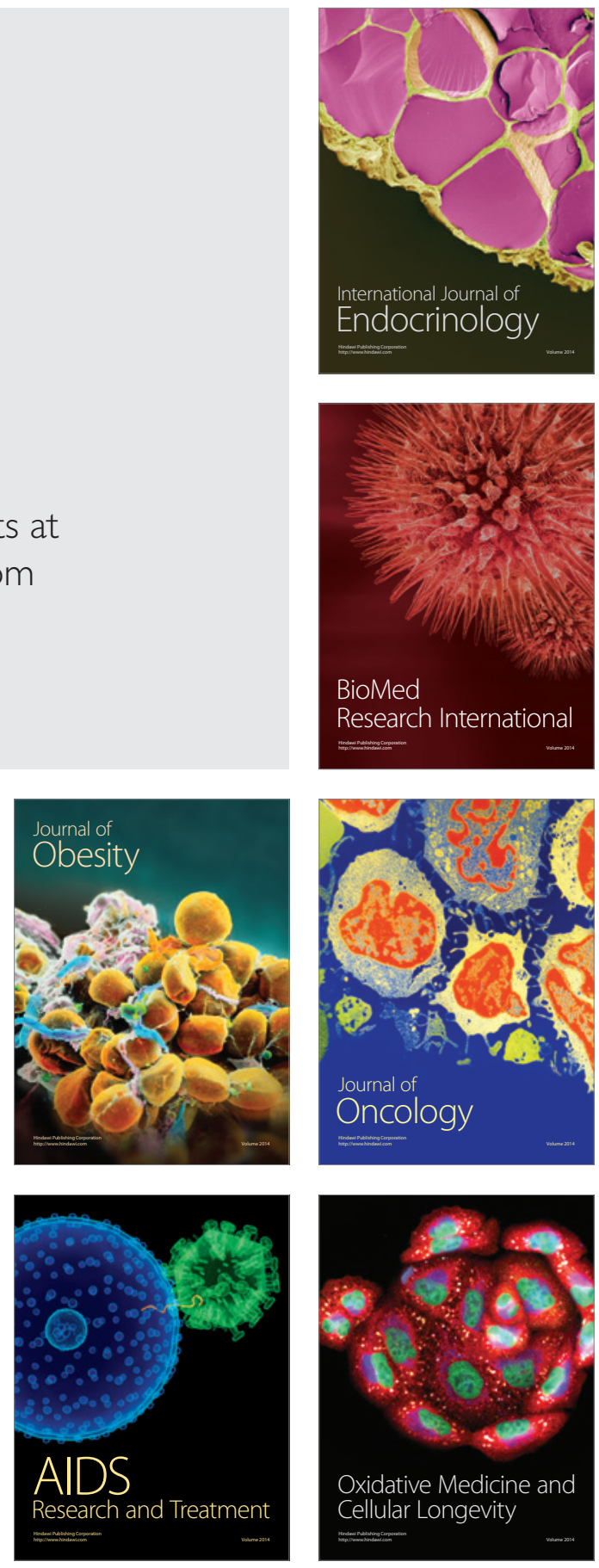